\title{
Stress-Softening Formulae of Polymer Bearings
}

\author{
M. H. B. M. Shariff, Anoop Sivasankaran, and Zanin Kavazović \\ Department of Applied Mathematics and Science, Khalifa University, P.O. Box 127788, Abu Dhabi, UAE
}

Correspondence should be addressed to Anoop Sivasankaran; anoop.sivasankaran@kustar.ac.ae

Received 1 July 2015; Accepted 28 September 2015

Academic Editor: Luigi Nicolais

Copyright (C) 2015 M. H. B. M. Shariff et al. This is an open access article distributed under the Creative Commons Attribution License, which permits unrestricted use, distribution, and reproduction in any medium, provided the original work is properly cited.

The motivation for this work was the absence of closed form solutions that can reasonably describe the axial deformation behaviour of stress-softening polymer bearings. In this paper, new closed form solutions that exhibit Mullins phenomenon are developed. We show that the apparent Young modulus depends on the shape factor and the minimal infinitesimal strain. We furthermore found that, in a nonlinear deformation, the shape factor plays an important role in stress softening. The solutions are design friendly and are consistent with expected results.

\section{Introduction}

When subjected to cyclic loadings, many polymers exhibit an anisotropic stress-softening phenomenon widely known as the Mullins effect (Mullins [1]). Recently, several researchers (Shariff [2-4]; Itskov et al. [5]; Dorfmann and Pancheri [6]; Merckel et al. [7]) developed anisotropic constitutive equations for stress-softening polymers. Shariff's 2014 model (Shariff [4]) compares well with different experimental data available in the literature for different types of rubberlike materials. In his 2014 model, Shariff [4] provides explicit theoretical results for homogeneous deformations. In this paper, however, we develop explicit closed form solutions for axial inhomogeneous deformation of polymer block bonded to two rigid plates. Polymer mounts have several applications and are, for instance, used in earthquake isolation bearings, in vehicle suspensions, and in bearings for bridges. The most important characteristic of a polymer mount is its load-deflection relation (stiffness). This property can be obtained experimentally or mathematically. However, for an axial compression (or tension) mounting, the deformation is significantly inhomogeneous. This results in great difficulties in mathematical treatment to obtain explicit closed form solutions. We note that explicit solutions have an advantage over numerical solutions, such as finite element solutions, in the sense that a numerical approach requires considerable computation effort to obtain specific values for design purposes. Since a numerical approach is not explicit in nature, it is not very convenient to use. In spite of these difficulties, approximate explicit closed form solutions have been developed in the past (Shariff [8-10]; Petrie and Shariff [11]; Haddow and Ogden [12]) by treating rubber as purely elastic. These approximate solutions predict fairly well the elastic mechanical behaviour of certain types of polymer (rubberlike) mounts. However, rubberlike materials are generally not purely elastic since they exhibit anisotropic stress-softening behaviour. Hence, the aim of this paper is to develop approximate explicit closed forms of solution for axial deformation of polymer mounts with Mullins's behaviour. The solutions are obtained without resorting to simplifying physical assumptions. Indeed, they are rather sought via a damage function and a variational principle. This leads to relatively simple theoretical solutions which are easy to handle and can be used as initial estimates for design purposes.

This paper is divided into seven sections. In Section 2, we define a useful concept of damage function. An average constitutive equation is given in Section 3 and it is used in a variational principle in Section 4. Approximate solutions are constructed in Section 5 and their results are discussed in Section 6. Section 7 concludes this paper.

\section{Isotropic Damage Function}

Before introducing the idea of a damage function, it is useful to present a few preliminaries needed in this paper. Throughout this document, all subscripts $i$ and $j$ take the 
values 1,2, and 3, unless otherwise stated. An eigenvalue (principal stretch) of the right stretch tensor $\mathbf{U}$ will be denoted by $\lambda_{i}$. The right Cauchy-Green tensor $C$ is given by $\mathbf{C}=\mathbf{U}^{2}=\mathbf{F}^{T} \mathbf{F}$, where $\mathbf{F}$ is the deformation tensor. In our case, incompressible polymers are considered; hence the relation $\lambda_{1} \lambda_{2} \lambda_{3}=1$ must be satisfied.

A measure of damage caused by strain is important in analysing stress-softening materials. Shariff [4] defined an anisotropic damage function to tackle anisotropic stresssoftening behaviour. However, in the current work, we are only dealing with average stress. Moreover, due to intractable mathematical analysis, isotropic damage is studied here. In view of this, we propose a damage function $g$ such that

$$
g(\mathbf{a})=\min _{\mathbf{u} \in S} g(\mathbf{u})
$$

where $S=\left\{\mathbf{u}=\left[u_{1}, u_{2}\right]^{T} \in R^{2}, u_{k}>0, k=1,2\right\}$. The function $g$ has also the properties that

$$
\widehat{g}^{\prime}(\alpha) \geq 0
$$

where

$$
\begin{aligned}
\widehat{g}(\alpha)= & g((1-\alpha) \mathbf{a}+\alpha \mathbf{b}), \\
& 0<\alpha \leq 1, \text { constant vectors } \mathbf{a}, \mathbf{b} \in S, \quad \mathbf{b} \neq \mathbf{a} .
\end{aligned}
$$

The first derivative $\widehat{g}^{\prime}(\alpha)$ may or may not exist at $\alpha=0$. If it exists, then $\hat{g}^{\prime}(0)=0$. In view of our definition, $g$ increases monotonically as $\mathbf{u}$ moves away in a straight line from the point $\mathbf{u}=\mathbf{a}$. It can be easily shown that

$$
I_{1}\left(\lambda_{1}, \lambda_{2}\right)=\operatorname{tr}(\mathbf{C})=\lambda_{1}^{2}+\lambda_{2}^{2}+\frac{1}{\lambda_{1}^{2} \lambda_{2}^{2}}
$$

(tr denotes the trace of a second-order tensor) is a damage function since it has only one extremum (global minimum) at the point $(1,1)$ for $\lambda_{1}>0$ and $\lambda_{2}>0$. In this paper, in order to obtain closed form solutions, we use the strain invariant $I_{1}$ as our damage function with $\mathbf{a}=[1,1]^{T}$; that is,

$$
g\left(\lambda_{1}, \lambda_{2}\right)=I_{1}\left(\lambda_{1}, \lambda_{2}\right) \text {. }
$$

We note that, throughout the history of the deformation, there exist $\lambda_{1 m}$ and $\lambda_{2 m}$ (not necessarily unique) such that

$$
g\left(\lambda_{1}, \lambda_{2}\right) \leq g\left(\lambda_{1 m}, \lambda_{2 m}\right)=\max _{0 \leq z \leq t} g\left(\lambda_{1}(z), \lambda_{2}(z)\right),
$$

where the material is subjected to a deformation history up to the current time $t$ and $z$ denotes a running time variable.

\section{Isotropic Model for the Mullins Effect}

In this study, we are only concerned with average stress softening as a first-order approximation. Moreover, we limit our study to isotropic stress softening in order to obtain explicit closed form expressions for the force-deflection relationships. Following the spirit of the work of Shariff [4] and Ogden and Roxburgh [14], we propose a "free" energy function $W_{f}$ of the form

$$
W_{f}=\eta W\left(\lambda_{1}, \lambda_{2}\right)+\phi\left(\eta, I_{m}\right),
$$

where $I_{m}=g\left(\lambda_{1 m}, \lambda_{2 m}\right)$ is a constant parameter and $W$ is a function that behaves similarly to a strain energy function of a purely elastic isotropic polymer. In this paper, we focus on moderate strain deformation and, in order to obtain closed form solutions and following the work of Shariff [9], we only consider the neo-Hookean form

$$
W\left(\lambda_{1}, \lambda_{2}\right)=\frac{\mu}{2}\left(I_{1}-3\right)
$$

where $\mu$ is the ground-state shear modulus of the virgin material.

The softening function $\eta$ is introduced to take into account the softening of the material under stress. We require (Ogden and Roxburgh [14]) expressing the softened Biot stress $\mathbf{T}^{(1)}=\partial W_{f} / \partial \mathbf{U}$, say, in the form

$$
\mathbf{T}^{(1)}=\eta \frac{\partial W}{\partial \mathbf{U}}+p \mathbf{U}^{-1}
$$

where $p$ is the Lagrange multiplier associated with the incompressible constraint; then we must impose the condition

$$
\frac{\partial \phi}{\partial \eta}\left(\eta, I_{m}\right)=-W\left(\lambda_{1}, \lambda_{2}\right)=-\frac{\mu}{2}\left(I_{1}-3\right) .
$$

In view of (10), $\eta$ depends on $I_{1}$ (damage function) and $I_{m}$ and has the softening property (Ogden and Roxburgh [14])

$$
0<\eta\left(I_{1}, I_{m}\right) \leq \eta\left(I_{m}, I_{m}\right)=1 \text {. }
$$

If we impose the condition that $W_{f}=0$ in the stress-free reference state, then we must have $\phi\left(\eta_{m}, I_{m}\right)=0$, where $\eta_{m}=\eta\left(3, I_{m}\right)$. To satisfy the Clausius-Duhem inequality, we impose the conditions $\partial \eta / \partial I_{m}<0$ and $\partial \phi / \partial I_{m}<0$. As we are only interested in evaluating the force-deflection expression and since the function $\phi$ does not appear in the stress-strain relationship such as (9), we will not elaborate on its properties here.

Figure 1 depicts nominal stress-strain paths for a simple tension deformation. The nominal stress on any path can be obtained by differentiating the area $W_{f}$ under an "elastic curve" path. On an elastic curve path, the value of $I_{m}$ is fixed. As a consequence, the "free" energy function $W_{f}$ for an inelastic solid can be portrayed by an infinite family of elastic strain energy functions parameterized by the internal variable $I_{m}$

In this paper, for simplicity, we consider

$$
\eta=e^{\beta\left(I_{1}-I_{m}\right)}
$$

where $\beta$ is a material constant. We could, of course, propose a more elaborate function for $\eta$, but it is not the purpose of this paper. In view of (10) to (12) and the condition $\phi\left(\eta_{m}, I_{m}\right)=0$, we have

$$
\begin{aligned}
& \phi\left(\eta, I_{m}\right) \\
& \quad=-\frac{\mu}{2}\left(\eta \ln (\eta)-\eta+\beta\left(I_{m}-3\right) \eta+e^{\beta\left(3-I_{m}\right)}\right) .
\end{aligned}
$$




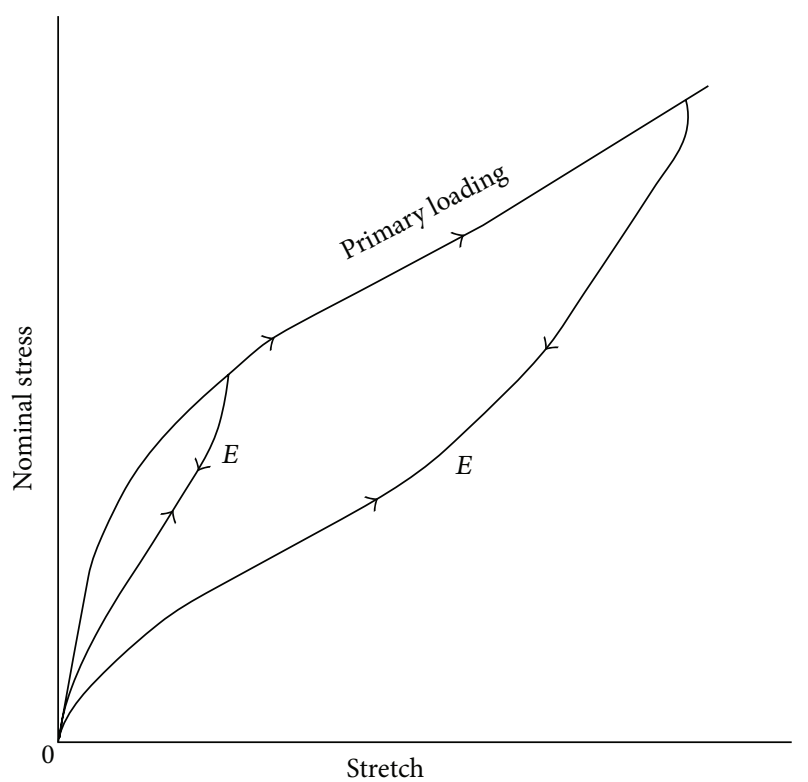

$\rightarrow \leftarrow E$ : elastic curve

FIGURE 1: Schematic loading-unloading curves in simple tension (Mullins effect).

3.1. Dissipation. The Clausius-Duhem inequality is given by the relation

$$
\text { DIS }=\operatorname{tr}\left(\mathbf{T}^{(1)} \dot{\mathbf{U}}\right)-\dot{W}_{f} \geq 0,
$$

where the superposed dot represents, for example, the time derivative. Note that

$$
\dot{W}_{f}=\operatorname{tr}\left(\frac{\partial W_{f}}{\partial \mathbf{U}} \dot{\mathbf{U}}\right)+\frac{\partial W_{f}}{\partial I_{m}} \dot{I}_{m} .
$$

In view of $\operatorname{det}(\mathbf{U})=1$ (det denotes the determinant of a tensor), we have

$$
\begin{aligned}
& \operatorname{tr}\left(\mathbf{U}^{-1} \dot{\mathbf{U}}\right)=0, \\
& \operatorname{tr}\left(\mathbf{T}^{(1)} \dot{\mathbf{U}}\right)=\operatorname{tr}\left(\left(\mathbf{T}^{(1)}-p \mathbf{U}^{-1}\right) \dot{\mathbf{U}}\right) .
\end{aligned}
$$

Hence, in view of (9) and (14), we have

$$
\text { DIS }=-\frac{\partial W_{f}}{\partial I_{m}} \dot{I}_{m}
$$

It is clear from the property of $g$ that $\dot{I}_{m} \geq 0$. The functional properties of $\eta$ and $\phi$ ensure that DIS $\geq 0$ which is consistent with the Clausius-Duhem inequality that indicates energy dissipation.

\section{A Variational Principle}

The equation of equilibrium of an incompressible material with negligible body force is

$$
\operatorname{Div} \mathbf{S}=\mathbf{0}, \quad \forall \mathbf{X} \in B_{0},
$$

where

$$
\mathbf{S}=\frac{\partial W}{\partial \mathbf{F}}+p \mathbf{F}^{-1}
$$

Div denotes the divergence operator relative to $B_{0}$, and $\mathbf{S}^{T}$ is the first Piola-Kirchhoff stress tensor.

The essential boundary condition is

$$
\zeta=\xi \quad \text { on } \partial B_{0}^{x}
$$

and the natural (traction) boundary condition on the stressfree surface is

$$
\mathbf{S}^{T} \mathbf{N}=\mathbf{0}, \quad \forall \mathbf{X} \in \partial B_{0}^{\sigma},
$$

where

$$
\begin{aligned}
& \partial B_{0}^{x} \cap \partial B_{0}^{\sigma}=\emptyset, \\
& \partial B_{0}^{x} \cup \partial B_{0}^{\sigma}=\partial B_{0},
\end{aligned}
$$

and $\mathbf{N}$ is the unit outward normal vector to $\partial B_{0}$.

When $I_{m}$ is fixed, $W_{f}$ can be treated as an isotropic elastic strain energy function. Equilibrium equation (18), traction boundary condition (21), and the constraint $J=1$ can be obtained via the first variation of the functional for an incompressible material

$$
\Pi(\zeta)=\int_{B_{0}}\left\{W_{f}+p(J-1)\right\} d V,
$$

where $\zeta(\mathbf{X})$ is any deformation field satisfying essential boundary condition (20) and $d V$ is the volume element appropriate to $B_{0}$. We note that $\zeta \in Y$, where $Y$ is a class of kinematically admissible deformation field defined by

$$
Y=\left\{\zeta \mid \zeta \in C^{2}\left(B_{0}\right), \zeta=\xi \text { on } \partial B_{0}^{x}\right\},
$$

where $C^{2}\left(B_{0}\right)$ is a class of twice continuously differentiable functions on $B_{0}$. The Lagrange multiplier $p$ belongs to the admissible class

$$
T=\left\{p \mid p \in C^{1}\left(B_{0}\right)\right\},
$$

where $C^{1}\left(B_{0}\right)$ is a class of differentiable functions on $B_{0}$. Indeed, vanishing of the first variation (23) yields

$$
\begin{aligned}
0= & \delta \Pi \\
= & \int_{B_{0}}\left\{\operatorname{tr}\left(\frac{\partial W_{f}}{\partial \mathbf{F}} \delta \mathbf{F}\right)+\delta p(J-1)+p \delta J\right\} d V \\
= & \int_{B_{0}}\{-\operatorname{Div} \mathbf{S} \cdot \delta \zeta+\delta p(J-1)\} d V \\
& +\int_{\partial B_{0}^{\sigma}} \mathbf{s}^{T} \mathbf{N} \cdot \delta \zeta d A,
\end{aligned}
$$

where $d A$ is the surface element appropriate to $\partial B_{0}$ and $\delta \zeta=$ 0 on $\partial B_{0}^{x}$. Since $\delta \zeta$ and $\delta p$ are arbitrary, then (18), (21), and $J=1$ follow by the calculus of variation, $\delta \zeta$ and $\delta p$ being interpreted as a variation in $\zeta$ and $p$, respectively. 


\section{Approximate Solution}

In the past, several researchers used simplifying physical assumptions in order to obtain approximate closed form solutions (Shariff [9], Gent and Lindley [15], and Klingbeil and Shield [16]). However, in this paper, we use the variational principle given in the previous section to obtain approximate explicit closed form solutions without resorting to facilitating physical assumptions. In this section, approximate solutions for axial deformations of bonded disc and rectangular strip are given. Figure 2 illustrates geometries of rectangular strip and bonded disc deformed under axial load. The approximate deformation solutions belong to the set $Y_{a} \subset Y$, where $Y_{a}=$ $\left\{\mathbf{u} \mid \mathbf{u} \in Y\right.$ and $\delta \mathbf{u}=\mathbf{g}$ on $\left.\partial B_{0}^{\sigma}\right\}$. Since $\delta \mathbf{u}$ is not arbitrary on $\partial B_{0}^{\sigma}$, traction-free condition (21) will not be satisfied exactly. However, it will be satisfied in a weighted sense. For instance, if $g$ is constant, then the zero traction on the free surface is approximated by zero average traction (see, e.g., Klingbeil and Shield [16]). Nevertheless, our solutions satisfy in an exact manner all three of the following: equilibrium equation (18), essential boundary condition (20), and incompressibility equation $J=1$.

5.1. Average Stress Softening. In the present study, we are mainly interested in calculating the force-deflection curve. To facilitate the evaluation of explicit closed form solutions, we use the average damage function $I_{a}$ (instead of $I_{1}$ ) in our calculation, where

$$
I_{a}(\alpha H)=\frac{1}{V\left(B_{0}\right)} \int_{B_{0}} I d V,
$$

where the parameter $\alpha H$ depends on the axial strain $h / H$ and

$$
V\left(B_{0}\right)= \begin{cases}2 \pi L^{2} H & \text { disc } \\ 4 L H & \text { strip }\end{cases}
$$

where the strip problem is considered as a plane strain problem. We note that $I_{a}$ is a damage function with properties given in Section 3. Instead of $W_{f}$ given in (7), we will use the free energy

$$
W_{a}=\eta\left(I_{a}, I_{a m}\right) W\left(\lambda_{1}, \lambda_{2}\right)+\phi\left(\eta, I_{a m}\right)
$$

in our calculation, where $I_{a m}=\max _{0 \leq z \leq t} I_{a}(\alpha H(z))$. The function $\phi$ in (29) is independent of the principal stretches $\lambda_{1}$ and $\lambda_{2}$.

5.2. Circular Cylindrical Disc. The geometry of the disc body is defined by

$$
\begin{aligned}
0 & \leq R \leq L, \\
-H & \leq Z \leq H, \\
0 & \leq \Theta \leq 2 \pi
\end{aligned}
$$

in the reference configuration, where $(R, \Theta, Z)$ are the cylindrical polar coordinates. Let $(r, \theta, z)$ denote corresponding coordinates in the deformed configuration. The material is

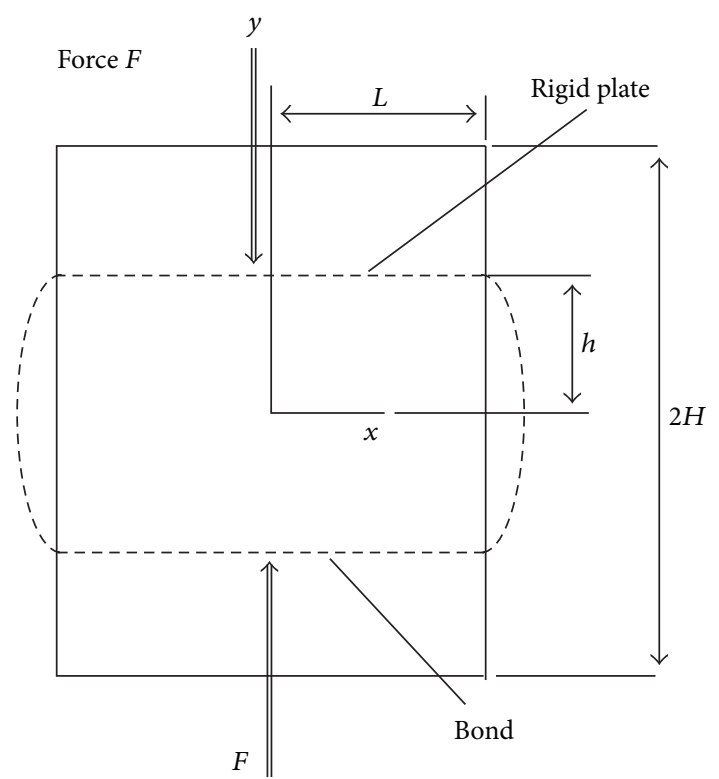

Plane strain rectangular strip

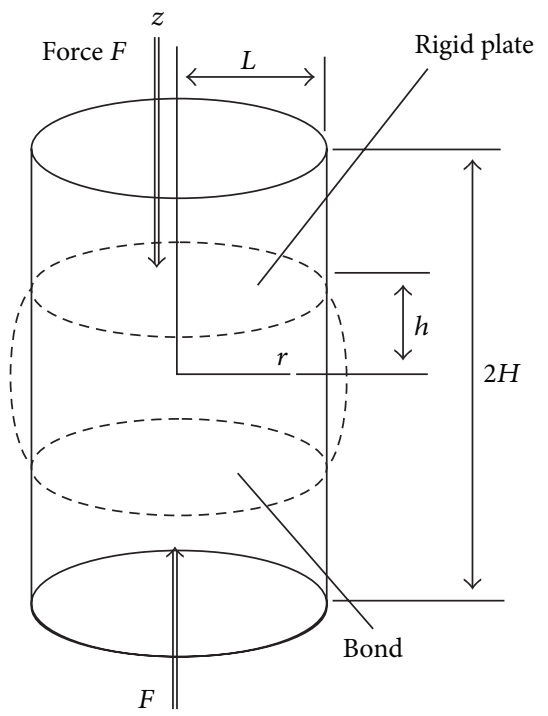

Cylindrical disc

FIGURE 2: Bonded mounts.

bonded to rigid plates at $Z=-H, H$. The essential boundary condition then takes the form

$$
\begin{aligned}
& r=R \quad \text { on } Z= \pm H, \\
& z= \pm h \quad \text { on } Z= \pm H .
\end{aligned}
$$

Following the work of Shariff [9], we use the following deformation field in the cylindrical problem:

$$
\begin{aligned}
& r=R f(Z), \\
& \theta=\Theta, \\
& z=g(Z),
\end{aligned}
$$


where

$$
\begin{aligned}
f(Z) & =f_{0} \cos (\alpha Z), \\
f_{0} & =f(0)=\sec (\alpha H), \\
g(Z) & =\frac{\tan (\alpha Z)}{f_{0}^{2} \alpha}
\end{aligned}
$$

for compression and

$$
\begin{aligned}
f(Z) & =f_{0} \cos (j \alpha Z), \\
f_{0} & =f(0)=\sec (j \alpha H), \\
g(Z) & =\frac{\tan (j \alpha Z)}{f_{0}^{2} j \alpha}
\end{aligned}
$$

for tension. The term $\alpha H$ is related to the axial strain via the equation

$$
\frac{h}{H}= \begin{cases}\frac{\sin (2 \alpha H)}{2 \alpha H} & \text { for compression } \\ \frac{\sin (2 j \alpha H)}{2 j \alpha H} & \text { for tension, }\end{cases}
$$

where $j=\sqrt{-1}$ and $0<\alpha H<\pi / 2$. Up to moderate strain (e.g., 30\%) $\alpha H<1$ and the right-hand side of (35) can be approximated using Taylor series up to the 4 th order and we can write $\alpha H$ explicitly in terms of $h / H$; that is,

$$
\alpha \begin{array}{ll}
\frac{\sqrt{10}}{2} \sqrt{1-\sqrt{1-\frac{6}{5}\left(1-\frac{h}{H}\right)}} & \text { for compression } \\
\frac{\sqrt{10}}{2} \sqrt{\sqrt{1-\frac{6}{5}\left(1-\frac{h}{H}\right)}-1} & \text { for tension. }
\end{array}
$$

5.2.1. Compression. It can be easily shown from (35) and (36) that $\alpha H$ is a monotonically decreasing function of $h / H$. The damage function invariant takes the form

$$
I_{1}=2 f^{2}(Z)+R^{2} f^{\prime 2}(z)+\frac{1}{f^{4}(Z)}
$$

and in view of (27), we have

$$
\begin{aligned}
I_{a}(\alpha H)= & 2 f_{0}^{2}\left(\frac{\sin (2 \alpha H)}{4 \alpha H}+\frac{1}{2}\right) \\
& +8\left(S \alpha H f_{0}\right)^{2}\left(\frac{1}{2}-\frac{\sin (2 \alpha H)}{4 \alpha H}\right) \\
& +\frac{1}{\alpha H f_{0}^{4}}\left(\tan (\alpha H)+\frac{\tan ^{3}(\alpha H)}{3}\right),
\end{aligned}
$$

where $S=L / 4 H$ is the shape factor (Shariff [9]) of the cylinder. From (38) it is clear that the shape factor plays a role in the average stress softening. With some algebra, we can show that $I_{a}^{\prime}(\alpha H)>0$ for the relevant range of $\alpha H$. This implies that $I_{a}$ is a monotonically increasing function of $\alpha H$ and hence it is a damage function with respect to the single variable $\alpha H$.

The physical components of the Cauchy stress relative to the $(r, \theta, z)$ system are

$$
\begin{aligned}
\frac{\sigma_{r r}}{\mu} & =\eta\left(I_{a}, I_{m}\right)\left(D+f_{0}^{2} \cos ^{2}(\alpha Z)-\frac{\sec ^{4}(\alpha Z)}{2 f_{0}^{2}}\right. \\
& \left.+\frac{f_{0}^{2} \alpha^{2} R^{2}}{2}\left(1+\sin ^{2}(\alpha Z)\right)\right), \\
\frac{\sigma_{\theta \theta}}{\mu} & =\eta\left(I_{a}, I_{m}\right)\left(D+f_{0}^{2} \cos ^{2}(\alpha Z)-\frac{\sec ^{4}(\alpha Z)}{2 f_{0}^{2}}\right. \\
& \left.+\frac{f_{0}^{2} \alpha^{2} R^{2} \cos ^{2}(\alpha Z)}{2}\right), \\
\frac{\sigma_{z z}}{\mu} & =\eta\left(I_{a}, I_{m}\right)\left(D+\frac{\sec ^{4}(\alpha Z)}{2 f_{0}^{2}}\right. \\
& \left.+\frac{f_{0}^{2} \alpha^{2} R^{2} \cos ^{2}(\alpha Z)}{2}\right), \\
\frac{\sigma_{r z}}{\mu} & =-\eta\left(I_{a}, I_{m}\right)\left(\frac{\alpha R \sec (\alpha Z) \tan (\alpha Z)}{f_{0}}\right) .
\end{aligned}
$$

The constant $D$ in (39) can be obtained using (26); that is,

$$
\int_{\partial B_{0}^{\sigma}} \mathbf{S}^{T} \mathbf{N} \cdot \delta \zeta d A=0
$$

since the left term of (26) vanishes. Note that our approximate solution $\zeta \in Y_{a}$, and hence $\delta \zeta=\mathbf{g}$ on the traction free surface. A different functional form of $\mathbf{g}$ will give a different value of $D$. As mentioned earlier in this section, if $\mathbf{g}$ is a constant, then our average traction on the free surface is zero. Alternatively, if we choose a value for $D$ (via some physical means), then seeking a specific functional form of $\mathbf{g}$ (so that (40) is satisfied) is not so important. In the current study, we choose a value of $D$ based on the work of Shariff [9]; that is,

$$
D=\frac{h}{H}-\frac{H^{2}}{h^{2}}-\frac{1}{2}-\left((\alpha H)^{2}+\gamma \alpha H \tan (\alpha H)\right) 4 S^{2},
$$

where $\gamma$ is an even function of the deformation $\alpha H$. For moderate strain, using Taylor's expansion, we have

$$
\gamma=1+\frac{\gamma^{\prime \prime}(0)}{2}(\alpha H)^{2}+\frac{\gamma^{(4)}(0)}{24}(\alpha H)^{4} .
$$

Note that neo-Hookean form (8) may not represent accurately some actual rubberlike materials. Hence, $\gamma$ can be used to compensate both the error of representing some rubberlike materials via the neo-Hookean form and error due to the approximations used in our model. Following the work of Shariff [9], we let $\gamma^{\prime \prime}(0)=2 / 3$ and $\gamma^{(4)}(0)=16$.

Let $A_{0}=\pi L^{2}$ be the area of the bonded surface and let $F$ be the normal force acting on the rigid plates. We then have

$$
\frac{F}{A_{0}}=\eta \frac{E}{3}\left(\frac{h}{H}-\left(\frac{H}{h}\right)^{2}-4 S^{2} \gamma \alpha H \tan (\alpha H)\right),
$$

where $E=3 \mu$ is the ground-state Young modulus. 
5.2.2. Tension. The results for tension are obtained from those for compression simply by replacing $\alpha$ by $j \alpha$.

5.3. Plane Strain Rectangular Strip. The geometry of the "infinite" strip in the $Z$ direction is defined by

$$
\begin{gathered}
-L \leq X \leq L, \\
-H \leq Y \leq H,
\end{gathered}
$$

where $(X, Y, Z)$ are the Cartesian coordinates in the reference configuration. If $(x, y, z)$ denote corresponding coordinates in the deformed configuration and the material is bonded to rigid plates at $Y=-H$ and $Y=H$, then the essential boundary condition takes the form

$$
\begin{aligned}
& x=X \quad \text { on } Y= \pm H, \\
& y= \pm h \quad \text { on } Y= \pm H .
\end{aligned}
$$

Following the work of Shariff [9], we use the following deformation field in the strip problem:

$$
\begin{aligned}
& x=x(X, Y), \\
& y=g(Y), \\
& z=Z .
\end{aligned}
$$

By symmetry $x(X, Y)$ is an odd function in $X$ and an even function in $Y$. In view of incompressibility condition, the essential boundary condition, and the relation

$$
\begin{array}{r}
x(0, Y)=0, \\
g(0)=0,
\end{array}
$$

we have

$$
x=X f(Y),
$$

where $f g^{\prime}=1$.

5.3.1. Compression. Following the work of Shariff [9], we have

$$
\begin{aligned}
& x=f_{0} X \cos (\alpha Y), \\
& y=\frac{\ln (\sec (\alpha Y)+\tan (\alpha Y))}{f_{0} \alpha},
\end{aligned}
$$

where

$$
\begin{aligned}
f_{0} & =f(0)=\sec (\alpha H), \\
\frac{h}{H} & =\frac{\cos (\alpha H) \ln (\sec (\alpha H)+\tan (\alpha H))}{\alpha H} .
\end{aligned}
$$

The damage functions take the form

$$
\begin{aligned}
I_{1}= & f^{2}+X^{2} f^{\prime 2}+\frac{1}{f^{2}}+1, \\
I_{a}= & f_{0}^{2}\left(\frac{\sin (2 \alpha H)}{4 \alpha H}+\frac{1}{2}\right) \\
& +\frac{4}{3}\left(S f_{0} \alpha H\right)^{2}\left(\frac{1}{2}-\frac{\sin (2 \alpha H)}{4 \alpha H}\right) \\
& +\frac{1}{f_{0}^{2}} \frac{\tan (\alpha H)}{\alpha H}+1,
\end{aligned}
$$

where the shape factor $S=L / 2 H$. The Cartesian components of the Cauchy stress are given by

$$
\begin{aligned}
& \frac{\sigma_{11}}{\mu}=\eta\left(I_{a}, I_{m}\right)\left(D+f_{0}^{2} \cos ^{2}(\alpha Y)-\frac{\sec ^{2}(\alpha Y)}{2 f_{0}^{2}}\right. \\
& \left.\quad+f_{0}^{2} \alpha^{2} X^{2} \frac{1+\sin (\alpha Y)}{2}\right), \\
& \frac{\sigma_{22}}{\mu}=\eta\left(I_{a}, I_{m}\right)\left(D+\frac{\sec ^{2}(\alpha Y)}{2 f_{0}^{2}}\right. \\
& \left.\quad+\frac{\left(f_{0} \alpha X \cos (\alpha Y)\right)^{2}}{2}\right), \\
& \frac{\sigma_{12}}{\mu}=-\eta\left(I_{a}, I_{m}\right) \alpha X \tan (\alpha Y), \\
& \frac{\sigma_{33}}{\mu}=\eta\left(I_{a}, I_{m}\right)\left(D-\frac{\sec ^{2}(\alpha Y)}{2 f_{0}^{2}}+\left(f_{0} \alpha X \cos (\alpha Y)\right)^{2}\right. \\
& \quad+2),
\end{aligned}
$$

where (Shariff [9])

$$
D=\frac{h}{H}-\frac{H^{3}}{h^{3}}-\frac{1}{2}-\frac{\left((\alpha H)^{2}+2 \gamma \alpha H \tan (\alpha H)\right) L^{2}}{6 H^{2}} .
$$

\section{Results}

In this section, we only discuss our results for the bonded disc. Results for disc tension and axial deformations of rectangular strip are similar, and we, hence, omit their discussions. It is clear from (38) that the softening function $\eta\left(I_{a}, I_{m}\right)$ depends on the shape factor $S$. Figure 3 shows the loaddeformation curves of the primary loading and "softened" elastic loading for $S=2.15$ and $E=17.5$ which behave as expected. Unfortunately, no stress-softening experiment for bonded polymers exists in the literature. Hence, we are not able to compare our theoretical results for various values of $S$. However, as $S$ approaches zero, the load-deflection behaviour (in tension) is equivalent to stretching a string (simple tension) and simple tension experiments do exist in the literature. In Figure 4, we compare our force-tension curves for $S=0$ with the experimental data of Mullins and Tobin [13]. The material used in the experiment was filler-reinforced GR-S tread vulcanizate rubber. It is clear in the figure that our theory compares well with the uniaxial experiment. We note that our theory is only developed for moderate strain.

For an infinitesimal deformation, the apparent Young modulus $E_{c}$ takes the simple form

$$
E_{c}=\left(1+e_{m}\right) E\left(1+2 S^{2}\right) \text {, }
$$




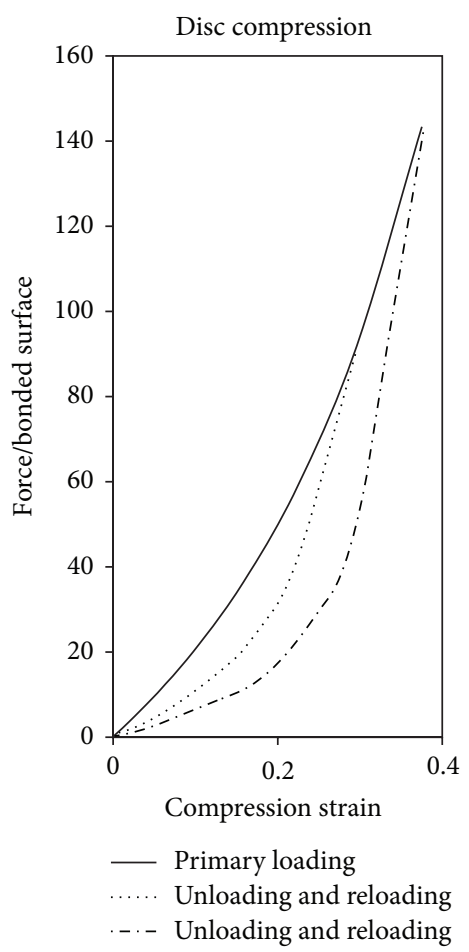

FIGURE 3: Load-compression curves of bonded disc.

where $e_{m}<0\left(\left|e_{m}\right| \ll 1\right)$ is the minimal infinitesimal strain. We note that $E_{c}$ is softened by the softening parameter $\left(1+e_{m}\right)$ which is independent of the shape factor $S$. "Purely elastic" $E_{c}$ can be easily recovered by letting $e_{m}=0$. Similarly, for the nonlinear case, the load-deflection curves of a purely elastic material can be easily recovered by letting $\eta=1$. Moreover, it is shown in Shariff [9] that these elastic curves compare well with experimental data of elastic rubberlike materials.

\section{Conclusion}

This work has demonstrated that explicit nonlinear (finite deformation) formulae may be obtained for axial deformations of bonded polymer (rubberlike) mounts with Mullins behaviour. The proposed formulae can be used as an initial approximation to facilitate design procedures since the forms of solution are relatively simple. Furthermore, the solutions satisfy all the governing equations exactly with the exception of the traction free surface where the governing equation is approximated in a weighted sense. The theoretical results obtained are consistent with expected behaviour. From our analysis, we found that the shape factor plays an important role in stress softening. This result is new and can not be found in the previous literature. In addition to the specific formulae developed and presented here, our work furthermore demonstrates an approach which may be adapted to other free energy functions. There is clearly a potential to apply our method to a wide variety of nonlinear polymeric materials that exhibit stress softening.

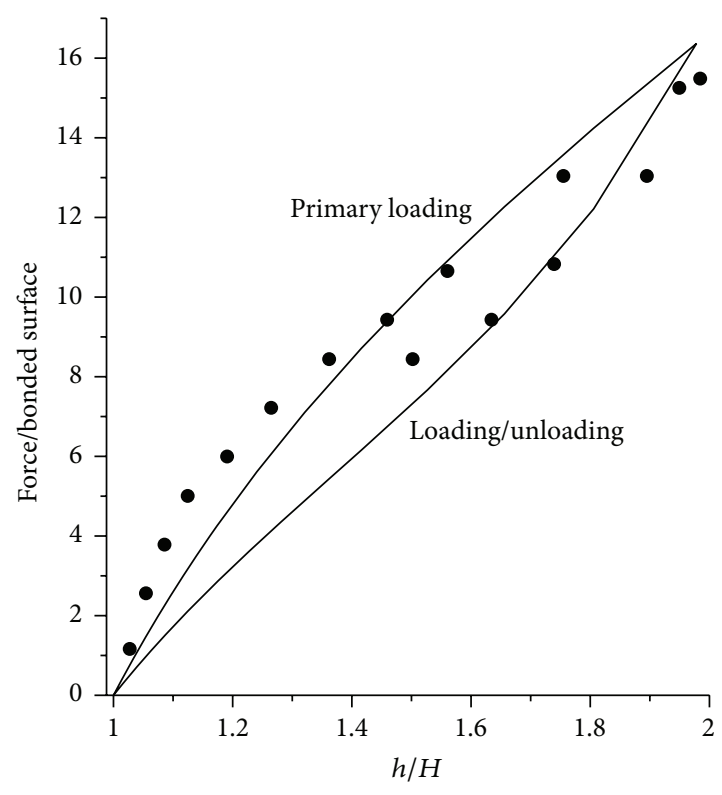

GR-S tread vulcanizate

- Experiment

- Theory

FIgUre 4: Comparison of theory with Mullins and Tobin [13] uniaxial experiment for $S=0 . E=28.5, \beta=0.04$.

\section{Conflict of Interests}

The authors declare that there is no conflict of interests regarding the publication of this paper.

\section{References}

[1] L. Mullins, "Effect of stretching on the properties of rubber," Journal of Rubber Research, vol. 16, pp. 275-289, 1947.

[2] M. H. B. M. Shariff, "An anisotropic model of the Mullins effect," Journal of Engineering Mathematics, vol. 56, no. 4, pp. 415-435, 2006.

[3] M. H. B. M. Shariff, "Anisotropic stress-softening model for compressible solids," Zeitschrift für angewandte Mathematik und Physik, vol. 60, no. 6, pp. 1112-1134, 2009.

[4] M. H. B. M. Shariff, "Direction dependent orthotropic model for Mullins materials," International Journal of Solids and Structures, vol. 51, no. 25-26, pp. 4357-4372, 2014.

[5] M. Itskov, A. E. Ehret, R. Kazakevičiute-Makovska, and G. W. Weinhold, "A thermodynamically consistent phenomenological model of the anisotropic mullins effect," Zeitschrift für Angewandte Mathematik und Mechanik, vol. 90, no. 5, pp. 370-386, 2010.

[6] A. Dorfmann and F. Q. Pancheri, "A constitutive model for the Mullins effect with changes in material symmetry," International Journal of Non-Linear Mechanics, vol. 47, no. 8, pp. 874-887, 2012.

[7] Y. Merckel, M. Brieu, J. Diani, and J. Caillard, "A Mullins softening criterion for general loading conditions," Journal of the Mechanics and Physics of Solids, vol. 60, no. 7, pp. 1257-1264, 2012. 
[8] M. H. B. M. Shariff, "An approximate analysis of infinitesimal deformations of bonded elastic mounts," The Journal of Strain Analysis for Engineering Design, vol. 23, no. 3, pp. 115-120, 1988.

[9] M. H. B. M. Shariff, "An analysis of non-linear deformation of bonded rubber mounts," Proceedings of the Institution of Mechanical Engineers. Part C. Journal of Mechanical Engineering Science, vol. 203, no. 2, pp. 113-119, 1989.

[10] M. H. B. M. Shariff, "A general approach to axial deformation of bonded elastic mounts of various cross-sectional shapes," Applied Mathematical Modelling, vol. 17, no. 8, pp. 430-436, 1993.

[11] C. J. S. Petrie and M. H. B. M. Shariff, "Finite deformation of compressible bonded rubber mounts," Journal of Strain Analysis for Engineering Design, vol. 27, no. 3, pp. 157-169, 1992.

[12] J. B. Haddow and R. W. Ogden, "Compression of bonded elastic bodies," Journal of the Mechanics and Physics of Solids, vol. 36, no. 5, pp. 551-579, 1988.

[13] L. Mullins and N. R. Tobin, "Theoretical model for the elastic behavior of filler-reinforced vulcanized rubbers," Rubber Chemistry and Technology, vol. 30, no. 2, pp. 555-571, 1957.

[14] R. W. Ogden and D. G. Roxburgh, "A pseudo-elastic model for the Mullins effect in filled rubber," Proceedings of the Royal Society of London A: Mathematical, Physical and Engineering Sciences, vol. 455, no. 1988, pp. 2861-2877, 1999.

[15] A. N. Gent and P. B. Lindley, "The compression of bonded rubber blocks," Proceedings of the Institution of Mechanical Engineers, vol. 173, no. 1, pp. 111-117, 1959.

[16] W. W. Klingbeil and R. T. Shield, "Large deformation analyses of bonded elastic mounts," Zeitschrift für angewandte Mathematik und Physik, vol. 17, no. 2, pp. 281-305, 1966. 

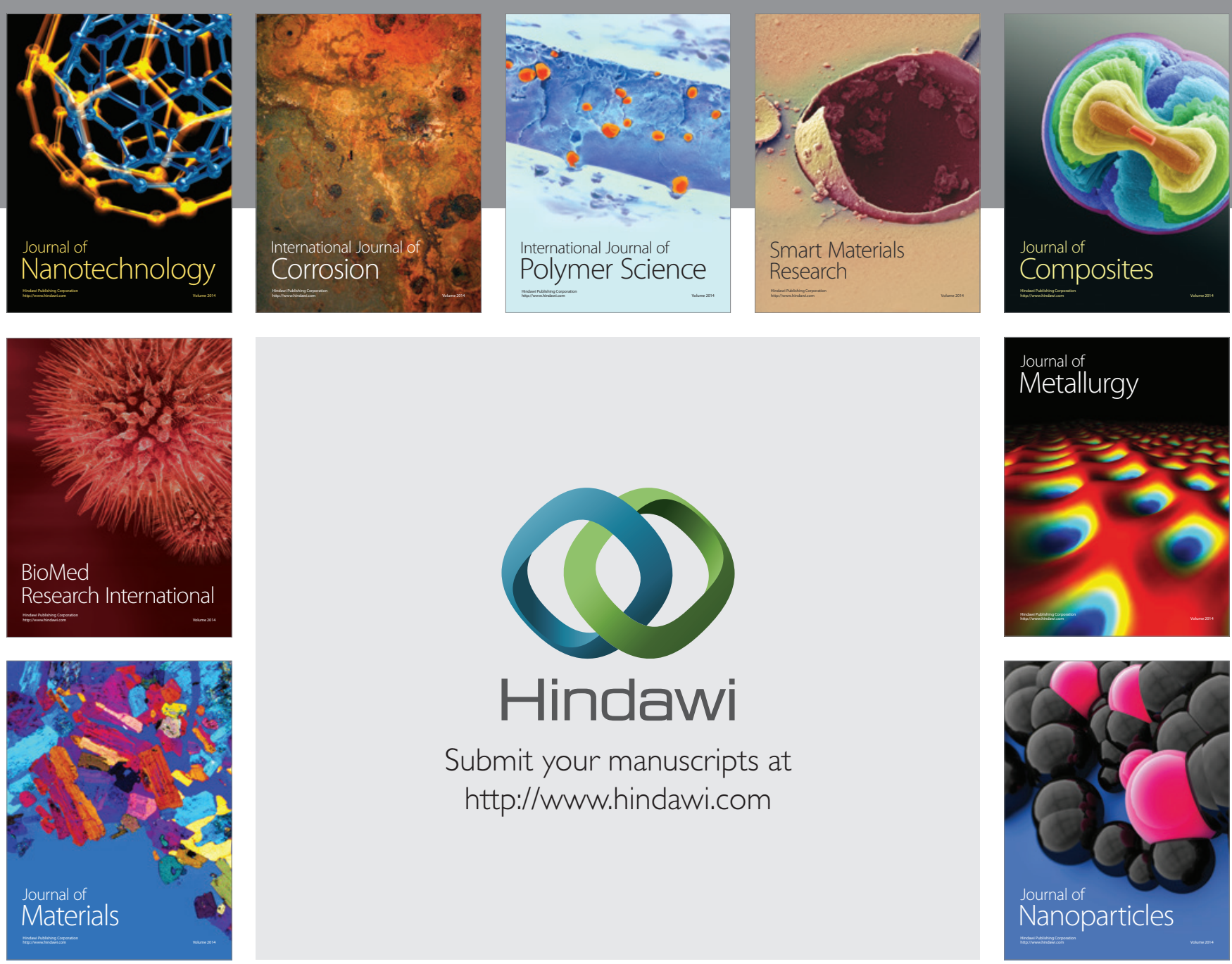

Submit your manuscripts at http://www.hindawi.com
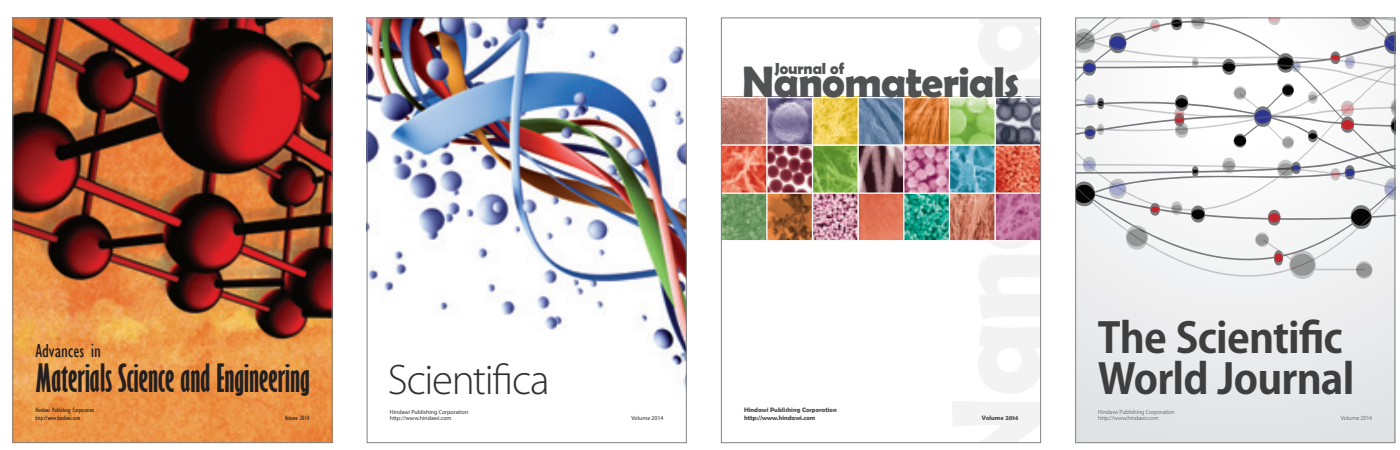

\section{The Scientific World Journal}
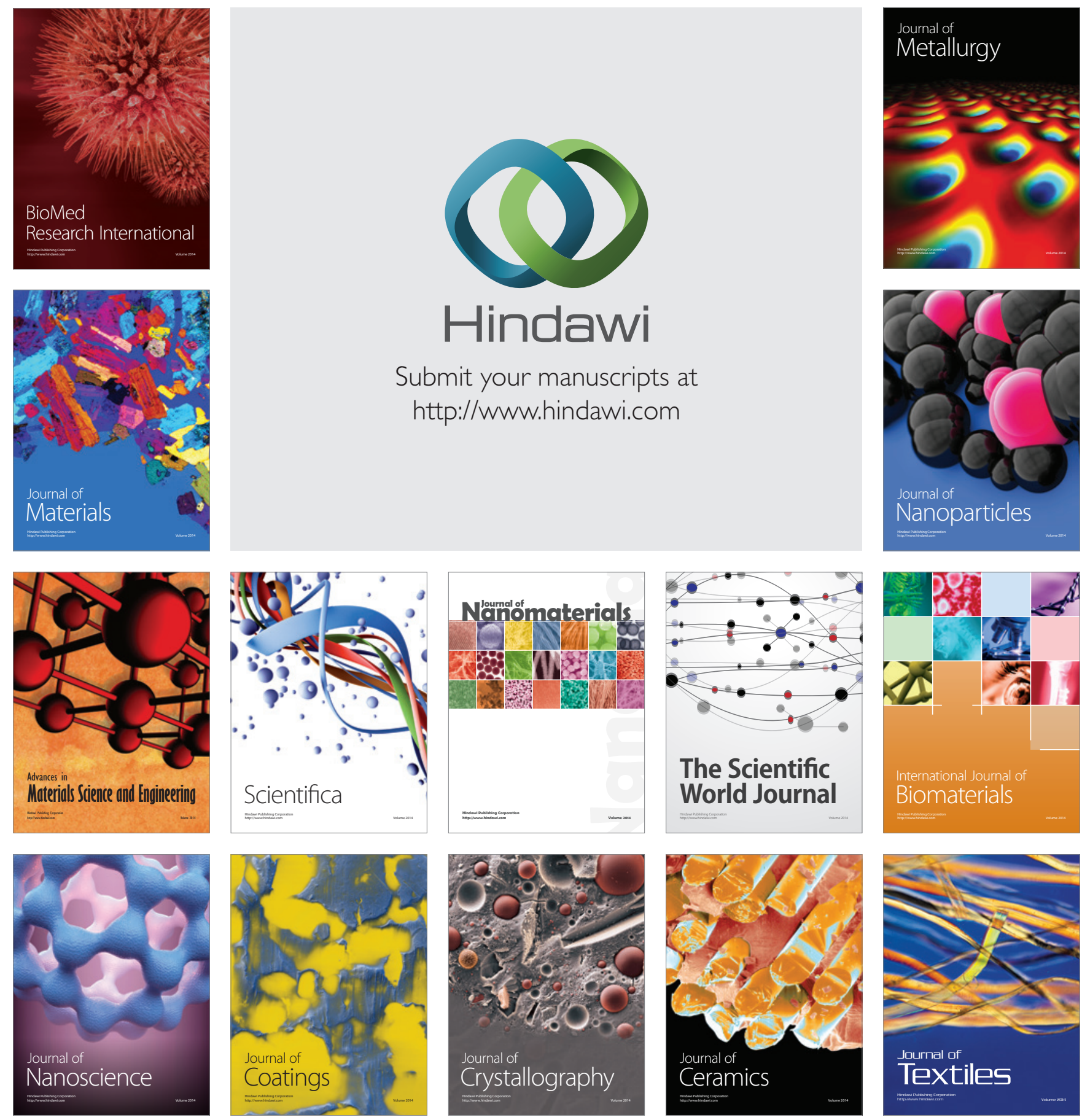